\title{
Cholesterol-Lowering Probiotics as Potential Biotherapeutics for Metabolic Diseases
}

\author{
Manoj Kumar, ${ }^{1}$ Ravinder Nagpal, ${ }^{2}$ Rajesh Kumar, ${ }^{1}$ R. Hemalatha, ${ }^{1}$ \\ Vinod Verma, ${ }^{3}$ Ashok Kumar, ${ }^{4}$ Chaitali Chakraborty, ${ }^{5}$ Birbal Singh, ${ }^{6}$ \\ Francesco Marotta, ${ }^{7}$ Shalini Jain, ${ }^{8}$ and Hariom Yadav ${ }^{9}$ \\ ${ }^{1}$ Department of Microbiology \& Immunology, National Institute of Nutrition, Hyderabad 50007, India \\ ${ }^{2}$ Shaheed Udham Singh College of Research \& Technology, Punjab, Mohali, Radaur, Haryana, India \\ ${ }^{3}$ Research and Development Unit, National Heart Centre, Singapore 1687521 \\ ${ }^{4}$ Department of Zoology, M.L.K. Post-Graduate College, Balrampur 271201, India \\ ${ }^{5}$ Department of Biotechnology, ITS Paramedical College, Ghaziabad 201206, India \\ ${ }^{6}$ Indian Veterinary Research Institute, Regional Station, Palampur 176061, India \\ ${ }^{7}$ Hepato-Gastroenterology Unit, S. Giuseppe Hospital, Vittore, 20123 Milano, Italy \\ ${ }^{8}$ Laboratory of Bioorganic Chemistry, National Institute of Diabetes and Digestive and Kidney Diseases, National Institutes of Health, \\ Bethesda, MD 20892, USA \\ ${ }^{9}$ Endocrinology, Diabetes, and Obesity Branch, National Institute of Diabetes and Digestive and Kidney Diseases, \\ National Institutes of Health, Bethesda, MD 20892, USA
}

Correspondence should be addressed to Shalini Jain, shalini2601@gmail.com and Hariom Yadav, yadavhariom@gmail.com

Received 18 October 2011; Accepted 10 January 2012

Academic Editor: Raffaele Marfella

Copyright (C 2012 Manoj Kumar et al. This is an open access article distributed under the Creative Commons Attribution License, which permits unrestricted use, distribution, and reproduction in any medium, provided the original work is properly cited.

Cardiovascular diseases are one of the major causes of deaths in adults in the western world. Elevated levels of certain blood lipids have been reported to be the principal cause of cardiovascular disease and other disabilities in developed countries. Several animal and clinical trials have shown a positive association between cholesterol levels and the risks of coronary heart disease. Current dietary strategies for the prevention of cardiovascular disease advocate adherence to low-fat/low-saturated-fat diets. Although there is no doubt that, in experimental conditions, low-fat diets offer an effective means of reducing blood cholesterol concentrations on a population basis, these appear to be less effective, largely due to poor compliance, attributed to low palatability and acceptability of these diets to the consumers. Due to the low consumer compliance, attempts have been made to identify other dietary components that can reduce blood cholesterol levels. Supplementation of diet with fermented dairy products or lactic acid bacteria containing dairy products has shown the potential to reduce serum cholesterol levels. Various approaches have been used to alleviate this issue, including the use of probiotics, especially Bifidobacterium spp. and Lactobacillus spp.. Probiotics, the living microorganisms that confer health benefits on the host when administered in adequate amounts, have received much attention on their proclaimed health benefits which include improvement in lactose intolerance, increase in natural resistance to infectious disease in gastrointestinal tract, suppression of cancer, antidiabetic, reduction in serum cholesterol level, and improved digestion. In addition, there are numerous reports on cholesterol removal ability of probiotics and their hypocholesterolemic effects. Several possible mechanisms for cholesterol removal by probiotics are assimilation of cholesterol by growing cells, binding of cholesterol to cellular surface, incorporation of cholesterol into the cellular membrane, deconjugation of bile via bile salt hydrolase, coprecipitation of cholesterol with deconjugated bile, binding action of bile by fibre, and production of short-chain fatty acids by oligosaccharides. The present paper reviews the mechanisms of action of anti-cholesterolemic potential of probiotic microorganisms and probiotic food products, with the aim of lowering the risks of cardiovascular and coronary heart diseases.

\section{Introduction}

Although cholesterol is an important basic block for body tissues, elevated blood cholesterol is a well-known major risk factor for coronary heart diseases [1]. WHO has predicted that, by 2030, cardiovascular diseases will remain the leading causes of death, affecting approximately 23.6 million people around the world [2]. It has been reported that 
hypercholesterolemia contributes to $45 \%$ of heart attacks in Western Europe and 35\% of heart attacks in Central and Eastern Europe [3]. The risk of heart attack is three times higher in those with hypercholesterolemia, compared to those who have normal blood lipid profiles. The WHO delineated that unhealthy diets, such as those high in fat, salt, and free sugar and low in complex carbohydrates, fruits, and vegetables, lead to increased risk of cardiovascular diseases [4]. Recent modalities for lowering blood cholesterol levels involve dietary management, behavior modification, regular exercise, and drug therapy [5]. Pharmacological agents that effectively reduce cholesterol levels are available for the treatment of high cholesterol; however, they are expensive and are known to have severe side effects [6]. Lactic acid bacteria (LAB) with active bile salt hydrolase (BSH) or products containing them have been suggested to lower cholesterol levels through interaction with host bile salt metabolism [7]. Lactobacilli with BSH activity have an advantage to survive and colonize the lower small intestine where the enterohepatic cycle takes place, and therefore BSH activity may be considered as an important colonization factor [8]. Sanders [9] proposed the mechanism based on the ability of certain probiotic lactobacilli and bifidobacteria to deconjugate bile acids enzymatically, increasing their rates of excretion. Cholesterol, being a precursor of bile acids, converts its molecules to bile acids replacing those lost during excretion leading to a reduction in serum cholesterol. This mechanism could be operated in the control of serum cholesterol levels by conversion of deconjugated bile acids into secondary bile acids by colonic microbes. The use of such orally applied microorganisms (probiotics) is a major aim of the concept of functional food $[10,11]$. Recently, there has been much interest in LAB, especially lactobacilli, due to their beneficial effects in health including anti-cholesterol, antidiabetic, antipathogenic, and anticarcinogenic properties and stimulation of the immune system [10, 12-19]. Lactobacillus plantarum, the predominating Lactobacillus species on oral and intestinal human mucosa, has shown the ability to survive the passage through the human gastrointestinal tract and to establish itself for at least a shorter time in the intestine after consumption [12, 16, 20].

Lactobacilli are frequently used in products for human consumption and can be found as probiotics in infant foods, cultured milks, and various pharmaceutical preparations $[10,21,22]$. One beneficial effect that has been suggested to result from human consumption of probiotic LAB is a reduction in serum cholesterol levels, as suggested by the results of several human and animal studies [23]. This effect can partially be ascribed to an enzymatic deconjugation of bile acids [24-27]. Deconjugated bile salts are less soluble and less efficiently reabsorbed from the intestinal lumen than their conjugated counterparts, which results in excretion of larger amounts of free bile acids in feces [28, 29]. Also, free bile salts are less efficient in the solubilization and absorption of lipids in the gut [30]. Therefore, the deconjugation of bile acids by LAB bacteria could lead towards a reduction in serum cholesterol either by increasing the demand of cholesterol for de novo synthesis of bile acids to replace that lost in feces or by reducing cholesterol solubility and,
TABLE 1: List of some potential bacteria showing bile salt hydrolase (BSH) activity.

\begin{tabular}{lc}
\hline Probiotic organisms with BSH activity & References \\
\hline Bifidobacterium adolescentis & {$[38]$} \\
B. animalis & {$[38]$} \\
B. breve & {$[38]$} \\
B. infantis & {$[39]$} \\
B. longum & {$[39]$} \\
Bifidobacterium sp. & {$[40,41]$} \\
Lactobacillus acidophilus & {$[41-43]$} \\
L. casei & {$[41-43]$} \\
L. fermentum & \\
L. gasseri & {$[38,41]$} \\
L. helveticus & {$[38]$} \\
L. paracasei subsp. paracasei & {$[44]$} \\
L. rhamnosus & {$[38,44]$} \\
L. plantarum & {$[8,19]$} \\
\hline
\end{tabular}

thereby, absorption of cholesterol throughout the intestinal lumen. Moreover, Gilliland et al. [31] observed a significant relationship between cholesterol assimilation by probiotic lactobacilli and their degree of bile deconjugation. BSH, the enzyme responsible for bile salt deconjugation during enterohepatic circulation, has been detected in several LAB species indigenous to the gastrointestinal tract (Table 1) [7, $28,32,33]$. It has also been suggested that $\mathrm{BSH}$ activity should be a requirement in the selection of probiotic organisms with cholesterol-lowering properties, as nondeconjugating organisms do not appear to be able to remove cholesterol from the culture medium to any significant extent [26]. Lactobacillus fermentum, a normal resident of the human gut microflora, has been reported to adhere to the epithelial cells, with a preference for the small intestine [34]. It has also been shown to colonize the intestine after oral administration [35] and produce surface-active components that inhibit the adhesion of uropathogenic bacteria $[36,37]$ (Table 2).

\section{Bile}

Bile is a yellow-green aqueous solution whose major constituents include bile acids, cholesterol, phospholipids, and the pigment biliverdin $[58,59]$. It is synthesized in the pericentral hepatocytes of the liver, stored and concentrated in the gallbladder interdigestively, and released into the duodenum after food intake. Bile functions as a biological detergent that emulsifies and solubilizes lipids, thereby playing an essential role in fat digestion. This detergent property of bile also confers potent antimicrobial activity, primarily through the dissolution of bacterial membranes [60,61]. Bile acids are saturated, hydroxylated C-24 cyclopentanophenanthrene sterols synthesized from cholesterol in hepatocytes. The two primary bile acids synthesized in the human liver are cholic acid (CA; 3a,7a,12a-trihydroxy-5b-cholan-24-oic acid) and 
TABLE 2: Summary of major findings for probiotic mediated cholesterol reduction.

\begin{tabular}{|c|c|c|c|c|}
\hline S. No. & Probiotic organism & Experimental system & Major findings & Reference \\
\hline 1 & Unknown (fermented milk) & Maasai tribesmen in Africa & Low cholesterol & {$[45]$} \\
\hline 2 & Unknown (Yogurt) & Human subjects & Reduced cholesterol & {$[46]$} \\
\hline 3 & Lactobacillus acidophilus & Culture media & $\begin{array}{l}\text { Cholesterol removal } \\
\text { Better survival in cholesterol } \\
\text { media }\end{array}$ & {$[31]$} \\
\hline 4 & Bifidobacterium & Culture media & Removal of cholesterol & {$[25]$} \\
\hline 5 & L. acidophilus & Culture media & Cholesterol assimilation & {$[47]$} \\
\hline 6 & Probiotic fermented milk & Rats & Cholesterol reducing efficacy & {$[48]$} \\
\hline 7 & L. reuteri & Mice & $\begin{array}{l}\text { Reduced blood cholesterol } \\
\text { Decreased triglycerides }\end{array}$ & {$[49]$} \\
\hline 8 & Bifidobacterium milk & Rats, Human & $\begin{array}{l}\text { Reduced cholesterol } \\
\text { Decreased triglyceride } \\
\text { Decreased LDL } \\
\text { Increased HDL }\end{array}$ & {$[50]$} \\
\hline 9 & $\begin{array}{l}\text { Yoghurt containing B. lactis or } \\
\text { B. longum }\end{array}$ & Rats & $\begin{array}{l}\text { Reduced cholesterol } \\
\text { Decreased triglyceride } \\
\text { Decreased LDL } \\
\text { Increased HDL }\end{array}$ & {$[51]$} \\
\hline 10 & L. plantarum & Culture media & Cholesterol assimilation & {$[52]$} \\
\hline 11 & L. bulgaricus and L. acidophilus & Human & Decreased cholesterol & Lin et al. [53] \\
\hline 12 & Lactobacillus sporogenes & Human & $\begin{array}{l}\text { Decreased Cholesterol } \\
\text { Reduced LDL-cholesterol }\end{array}$ & {$[54]$} \\
\hline 13 & L. acidophilus & Human & Decreased cholesterol & Gilliland [55] \\
\hline 14 & E. faecium & Human & $\begin{array}{l}\text { Decreased cholesterol levels } \\
\text { Decreased triglyceride } \\
\text { Decreased LDL } \\
\text { Increased HDL }\end{array}$ & {$[56]$} \\
\hline 15 & $\begin{array}{l}\text { Microencapsulated bile salt } \\
\text { hydrolase- (BSH-) active } \\
\text { Lactobacillus reuteri NCIMB } 30242\end{array}$ & Human & $\begin{array}{l}\text { Reduced LDL-cholesterol } \\
\text { Decreased total } \\
\text { cholesterol } \\
\text { Decreased apoB-100 } \\
\text { Decreased } \\
\text { non-HDL-cholesterol }\end{array}$ & {$[57]$} \\
\hline
\end{tabular}

chenodeoxycholic acid (CDCA; 3a,7a-dihydroxy-5b-cholan24-oic acid). Bile acids are further metabolized by the liver via conjugation ( $\mathrm{N}$-acyl amidation) to glycine or taurine, a modification that decreases the Pka to approximately 5 . Thus, at physiological $\mathrm{pH}$, conjugated bile acids are almost fully ionized and may be termed bile salts [62]. The primary bile acids, cholic and chenodeoxycholic acid, are synthesized de novo in the liver from cholesterol. The solubility of the hydrophobic steroid nucleus is increased by conjugation as an $\mathrm{N}$-acyl amidate with either glycine (glycoconjugated) or taurine (tauroconjugated) prior to secretion. The resulting molecules are therefore amphipathic and can solubilize lipids to form mixed micelles. Bile acids are efficiently conserved under normal conditions by a process termed enterohepatic recirculation. Conjugated and unconjugated bile acids are absorbed by passive diffusion along the entire gut and by active transport in the terminal ileum [58]. Reabsorbed bile acids enter the portal bloodstream and are taken up by hepatocytes, reconjugated, and resecreted into bile. Approximately $5 \%$ of the total bile acid pool
(0.3 to $0.6 \mathrm{~g}$ ) per day eludes epithelial absorption and may be extensively modified by the indigenous intestinal bacteria [63]. One important transformation is deconjugation, a reaction that must occur before further modifications are possible [64]. Deconjugation is catalyzed by BSH enzymes (EC 3.5.1.24), which hydrolyze the amide bond and liberate the glycine/taurine moiety from the steroid core (Figure 1). The resulting acids are termed unconjugated or deconjugated bile acids.

\subsection{Identification of bsh Homologs in Probiotic Genomes.} The genes that may encode BSH enzymes in the genome sequences of potential probiotic bacteria are available in public databases (National Center for Biotechnology Information genome site (http://www.ncbi.nlm.nih.gov/) and the Joint Genome Institute microbial genomics site (http://genome.jgi-psf.org/)). Several strains (e.g., Lactobacillus plantarum WCFS1) possess more than one BSH homolog, which are not identical. The genetic geography of $b s h$ regions is not the same in all strains, and in cases where 


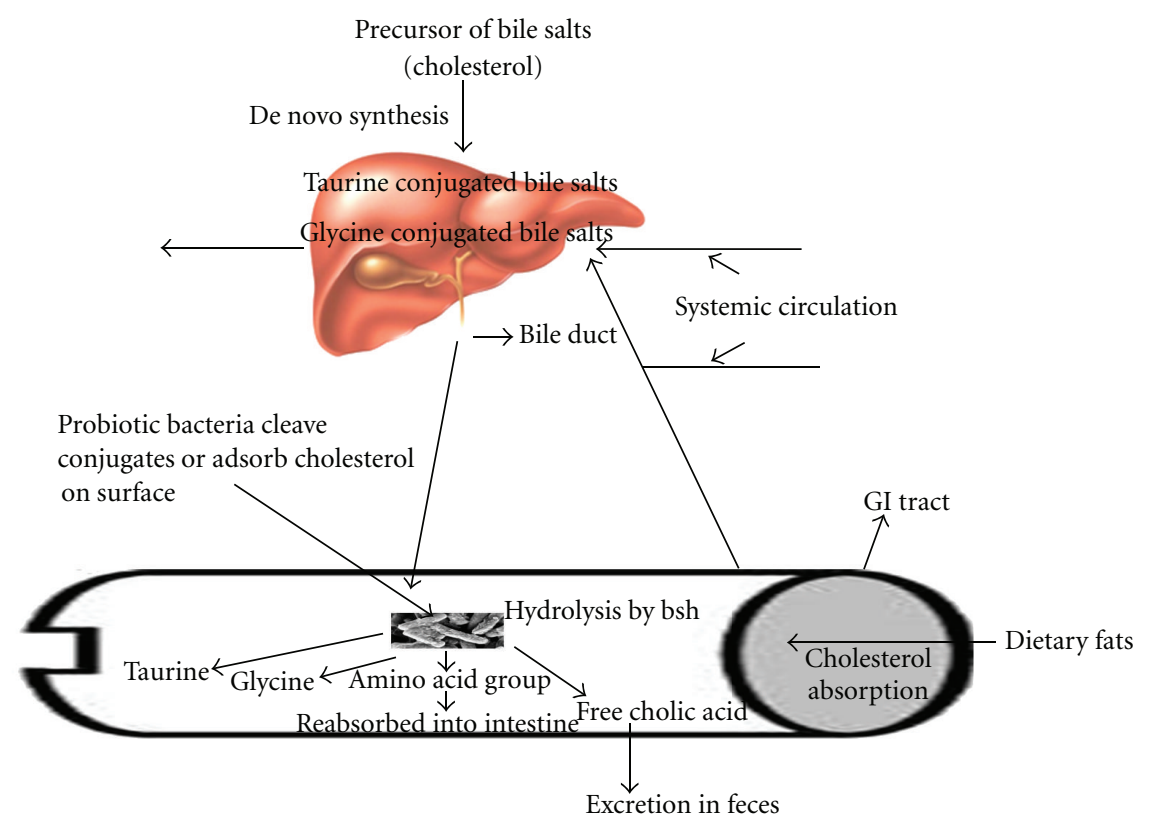

FIgURE 1: Cholesterol as the precursor for the synthesis of new bile acids and the hypocholesterolemic role of bile salt hydrolase (BSH).

more than one is present they are not located in the same region of the chromosome.

2.2. bsh Genes in Probiotic Bacteria. Since variability in bsh phenotypes has been observed within isolates of some species $[38,42,65,66]$, it has been speculated that bsh genes may have been acquired horizontally [65]. Comparison of the $b s h$ gene and surrounding sequences of $L$. acidophilus strain KS-13 and L. johnsonii 100-100 by Elkins et al. [65] has revealed little or no synteny flanking this locus. It was also noted that L. johnsonii 100-100 encodes a group II intron protein (maturase mat) downstream of $b s h$. In addition to reverse transcriptase activity, these proteins can function as maturases and endonucleases and facilitate movement and splicing of cDNA into the genome. Group II intron proteins are often inserted in or associated with mobile genetic elements [67]. Sequencing of the entire genome of L. acidophilus NCFM has revealed that this strain possesses two $b s h$ genes ( $b \operatorname{sh} A$ and $b \operatorname{sh} B$ ). The predicted sequence of the BSH enzymes encoded by these loci share a higher level of similarity to BSH enzymes from other Lactobacillus species than to each other, suggesting that they may have been acquired from different sources [68]. In short, $\mathrm{BSH}$ is present in all bifidobacterial strains and lactobacilli strains associated with the gastrointestinal environment, but $b s h$ genes can potentially be acquired from these strains by other intestinal microorganisms (e.g., L. monocytogenes).

\section{Functions of BSH}

The precise function(s) of microbial BSHs is currently not understood, although several hypotheses have been proposed, as follows.
3.1. Nutritional Role. The amino acids liberated from bile salt deconjugation could potentially be used as carbon, nitrogen, and energy sources, since glycine may be metabolized to ammonia and carbon dioxide, and taurine may be metabolized to ammonia, carbon dioxide, and sulfate. Bile salt deconjugation may therefore confer a nutritional advantage on hydrolytic strains. In support of this hypothesis, Huijghebaert et al. [69] and Van Eldere et al. [70] observed that certain BSH-positive strains of Clostridium utilized the released taurine as an electron acceptor, and growth rates were improved in the presence of taurine and taurine-conjugated bile salts. It has also been noted that transcription of the Bifidobacterium longum bsh gene is coupled to a homolog of $g \ln E$ that encodes a glutamine synthetase adenyltransferase that forms part of the nitrogen regulation cascade [39]. However, experiments performed by Tannock et al. [71] and Gilliland and Speck [72] refute this hypothesis since these authors observed that the lactobacilli used in their studies did not utilize the steroid moiety of the bile salt for cellular precursors since neither ring cleavage nor subsequent metabolism occurred.

3.1.1. Alteration of Membrane Characteristic. The bacteriolytic enzymes lysozyme and phospholipase A2, and antimicrobial peptides such as $\alpha$-defensins, are important contributors to innate immunity in the intestine. The composition, fluidity, permeability, hydrophobicity, and net charge of bacterial membranes all determine the extent of damage by these host defenses. It has been proposed that BSHs facilitate incorporation of cholesterol or bile into bacterial membranes [73-75]. This incorporation may increase the tensile strength of the membranes [76] or may change their fluidity or charge. Cell surface modifications that may result from BSH activity could potentially offer 
protection against perturbation of the structure and integrity of bacterial membranes by the immune system, and such resistance mechanisms may be important in establishing persistent infections. Such a function may strongly select for commensals possessing BSH enzymes while mitigating against BSH-negative pathogens or other transients.

3.1.2. Bile Detoxification. Studies by various research groups using wild-type and $b s h$ mutant pairs have provided a link between bile salt hydrolysis and bile tolerance. A Lactobacillus amylovorus mutant with a partial decrease in BSH activity isolated using an $\mathrm{N}$-methyl-N1-nitro- $N$-nitrosoguanidine mutagenesis strategy displayed decreased growth rates in the presence of bile salts [40]. Also, mutation of bsh in Lactobacillus plantarum [8] and Listeria monocytogenes [60, 61 ] renders cells significantly more sensitive to bile and bile salts. The precise mechanism by which $\mathrm{BSH}$ enzymes play a role in the tolerance of bile is not yet fully understood. However, it has been proposed that since the protonated (non-dissociated) form of bile salts may exhibit toxicity through intracellular acidification in a manner similar to organic acids, BSH-positive cells may protect themselves through the formation of the weaker unconjugated counterparts [77]. This could help negate the drop in $\mathrm{pH}$ by recapturing and exporting the cotransported proton. The ratio of glycoconjugated to tauroconjugated bile salts in human bile is usually $3: 1$. In vitro experiments have revealed that whereas tauroconjugated bile salts usually only have slight affects (if any) on bacterial cells at every $\mathrm{pH}$ examined, glycoconjugated bile salts are extremely toxic at acidic $\mathrm{pHs}$, and $b s h$ mutants are significantly more inhibited than corresponding parent cells $[60,61,77]$. Therefore, it has been suggested that BSHs are particularly important in combating the toxic effects of glycoconjugated bile salts at low $\mathrm{pH}$, and BSH activity may be of particular importance at the point where bile enters the duodenum and where acid reflux may occur from the stomach or in localized microenvironments in the intestine when the $\mathrm{pH}$ is lowered by lactic acid bacteria. The fact that BSHs have been shown to preferentially hydrolyze glycoconjugated bile salts $[78,79]$, together with the observation that BSHs have slightly acidic $\mathrm{pH}$ optima (usually between $\mathrm{pH} 5$ and 6) $[42,80]$, may serve to substantiate this theory.

3.1.3. Gastrointestinal Persistence. Since BSHs may combat the deleterious effects of bile (and perhaps components of the innate immune system such as the defensins through cell surface modifications), a role for these enzymes in survival/persistence of strains within the gastrointestinal tract is conceivable. Bateup et al. [81] compared the abilities of three Lactobacillus strains which demonstrated various degrees of BSH activity in vitro (one strain demonstrated high activity, one showed moderate activity, and one lacked activity) to colonize Lactobacillus-free mice. Enumeration of lactobacilli in the gastrointestinal organs 2 weeks after inoculation revealed that all strains colonized equally well, leading to the conclusion that $\mathrm{BSH}$ is not essential for colonization. However, a more recent study by Dussurget et al. [82] convincingly demonstrates that BSH contributes to persistence of L. monocytogenes within the gastrointestinal tract. A bsh mutant demonstrated reduced bacterial fecal carriage after oral infection of guinea pigs (counts of the mutant were 4 to 5 logs lower than the parent after $48 \mathrm{~h}$ ). It was also observed that intestinal multiplication of the parent could be increased approximately 10-fold by supplying cells with an extra copy of the gene on a plasmid, further confirming the importance of BSH to intestinal persistence [82]. Two obvious differences between this L. monocytogenes study and the earlier one of Bateup et al. [81] may account for their different conclusions. First, isogenic L. monocytogenes wild-type and bsh mutant strains were compared, and it is possible that intrinsic differences between the strains of lactobacilli used in the other study masked the contribution of BSH to intestinal survival. Furthermore, Bateup et al. [81] used Lactobacillusfree mice, and it is possible that a role for BSH would be uncovered in a more competitive environment. Therefore, future investigations with bifidobacterial and Lactobacillus $b s h$ mutants would be necessary to unequivocally determine whether gastrointestinal persistence is a universal function of BSHs.

\subsection{Impact of Microbial BSH Activity on the Host}

3.2.1. Cholesterol Lowering. Hypercholesterolemia (elevated blood cholesterol levels) is considered a major risk factor for the development of coronary heart disease, and although pharmacologic agents are available to treat this condition (e.g., statins or bile acid sequestrants), they are often suboptimal and expensive and can have unwanted side effects [83]. Oral administration of probiotics has been shown to significantly reduce cholesterol levels by as much as 22 to $33 \%[7,23]$ or prevent elevated cholesterol levels in mice fed a fat-enriched diet [84]. These cholesterol-lowering effects can be partially ascribed to BSH activity (other possible mechanisms include assimilation of cholesterol by the bacteria, binding of cholesterol to the bacterial cell walls, or physiological actions of the end products of shortchain fatty acid fermentation (Figure 2)) [80]. Deconjugated bile salts are less efficiently reabsorbed than their conjugated counterparts, which results in the excretion of larger amounts of free bile acids in feces. Also, free bile salts are less efficient in the solubilization and absorption of lipids in the gut. Therefore, deconjugation of bile salts could lead to a reduction in serum cholesterol either by increasing the demand for cholesterol for de novo synthesis of bile acids to replace those lost in feces or by reducing cholesterol solubility and thereby absorption of cholesterol through the intestinal lumen.

Impaired Digestive Functions. Since unconjugated bile acids are less efficient than conjugated molecules in the emulsification of dietary lipids and the formation of micelles, BSH activity may compromise normal lipid digestion and the absorption of fatty acids and monoglycerides could be 


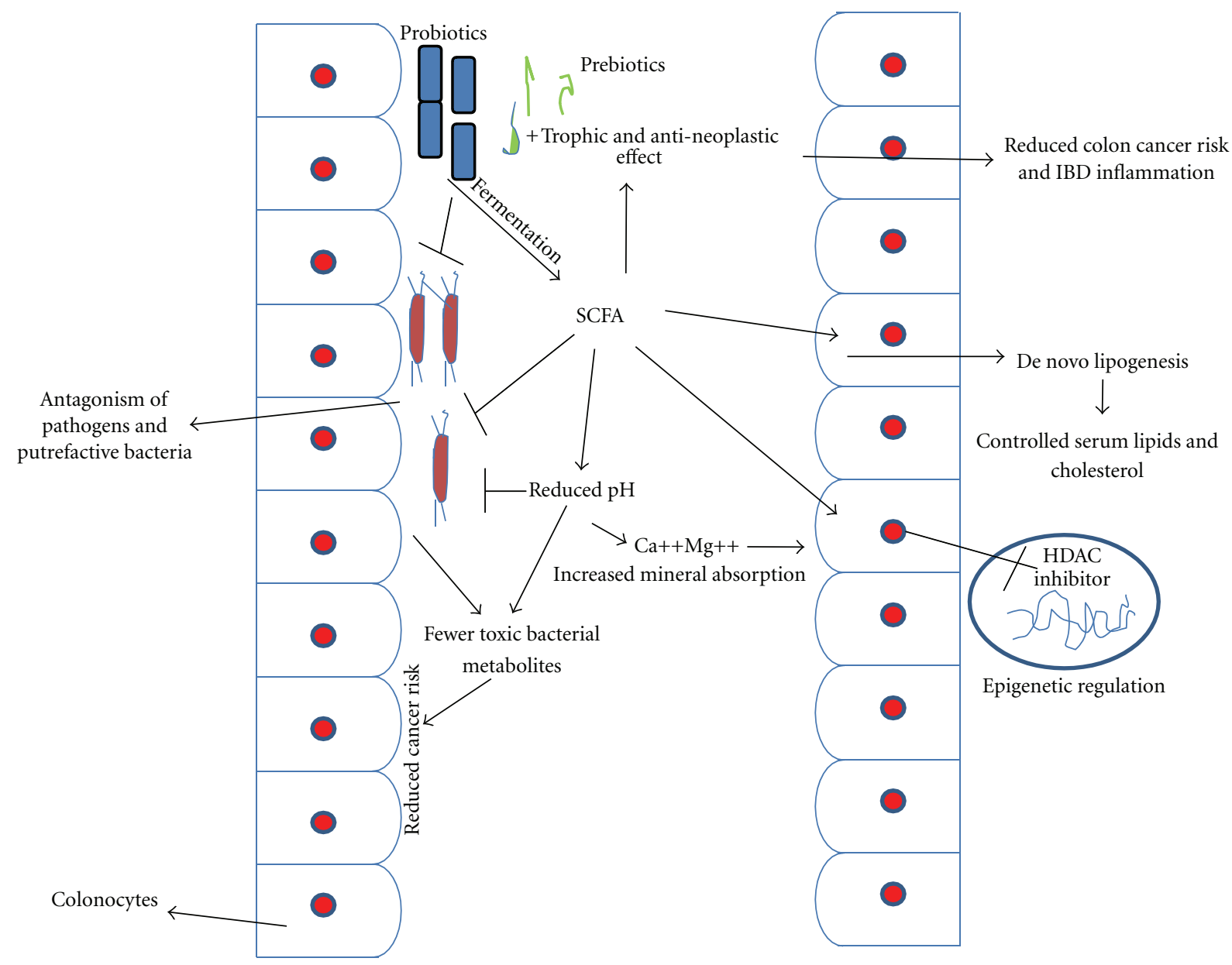

FIGURE 2: Role of probiotics' metabolites as epigenetic approach to control high cholesterol and colon cancer.

impaired [28]. Microbial BSH activity has been related to growth defects in chickens [85] but not in mice [81].

\section{Effects of Probiotics on Plasma Lipids}

The idea about the health advantages of fermented milk products in humans goes back to the early 19th century, when it was proposed by Metchnikov that fermenting milks by lactic acid bacteria "prevented intestinal putrefaction" and "helped maintain the forces of the body" $[22,86]$. A study on Maasai tribesmen in Africa who have low serum cholesterol showed that they rarely experience coronary heart diseases, despite eating a great deal of meat. They regularly consumed 4-5 liters of fermented whole milk per day. This provided the motivation for investigating fermented milk's possible influence on blood cholesterol [45]. Later, in a study by Mann [46], on twenty-six volunteers, it was found that large amount of yoghurt reduced cholesterolemia, which could be due to a factor in yoghurt that prevents production of cholesterol from acetate. This factor may be either orotic acid or 3-hydroxy-3-methylglutaric acid plus thermophilus milk or methanol soluble of thermophilus milk. Gilliland et al. [31] showed that some strains of Lactobacillus acidophilus make it possible for cholesterol to be bound to intestine's lumen and as a result decrease its absorption. Tahri et al. [25] investigated assimilation of cholesterol by Bifidobacterium strains and observed that the removal of cholesterol from the growth medium is caused by both bacterial activity and precipitation of cholesterol. Lin and Chen [47] investigated cholesterol-reducing abilities of L. acidophilus and found that hypocholesterolemic ability is because of the assimilation of cholesterol by $L$. acidophilus cells or its attachment to the surface of L. acidophilus cells. Grunewald [48] observed a strong reduction in serum cholesterol of probiotic fermented milk-fed rats, indicating that cholesterol level in serum can be reduced by consumption of probiotics. A different study conducted on mice with high cholesterol demonstrated that L. reuteri was able to reduce blood triglyceride by $38 \%$ and cholesterol by $40 \%$ and raised the HDL/LDL cholesterol ratio by $20 \%$ [49]. Xiao et al. [50] also observed that consumption of Bifidobacterium milk leads to a meaningful reduction in triglyceride, low-density lipid, and total cholesterol. Similar results were observed by Abd El-Gawad et al. [51] in a study on rats fed with yoghurt containing B. lactis or $B$. longum. Recently, Kumar et al. [52] also explored probiotic L. plantarum with potential to control hypercholesterolemia. Lin et al. [53] conducted human studies and observed that blood cholesterol was reduced significantly in volunteers 
who were given tablets of L. bulgaricus and L. acidophilus for 16 weeks every day. In another study, hyperlipidemic patients that were given Lactobacillus sporogenes for 90 days showed a $35 \%$ and $32 \%$ decrease in their LDL and total cholesterol levels, respectively [54]. The result by Anderson and Gilliland [87] showed a significant decrease $(2.4 \%)$ in blood cholesterol for fermented milk containing $L$. acidophilus during controlled clinical trials. In a randomized clinical trial on yoghurt starters plus Bifidobacterium longum, participants showed a decrease in total cholesterol [50]. In a randomized, double-blind, placebo-controlled clinical trial, it was demonstrated that E. faecium probiotic strain reduced cholesterol levels by $12 \%$. Klein et al. [56] also observed a significant reduction (11.6\%) in serum triglyceride levels during the period probiotics consumption in a placebocontrolled, double-blind, randomized crossover study. More recently, Jones et al. [57] reported that yoghurt formulation containing microencapsulated bile salt hydrolase- (BSH-) active Lactobacillus reuteri NCIMB 30242 is efficacious and safe for lowering LDL-C, TC, apoB-100, and non-HDL-C in hypercholesterolaemic subjects. Altogether, findings from in vitro systems and animal studies as well as human trails strongly suggest that probiotics have potential to ameliorate the cholesterol metabolic dysfunction, especially mediated through BSH activity and other unknown mechanisms, but the exact mechanism(s) of action for probiotics' mediated decrease in cholesterol levels are not completely known. Here, before discussing the probiotics' mechanism of action on plasma lipids, a summary of lipoprotein synthesis and metabolism is reviewed, as follows for better understanding by general readers the point of actions of probiotics on lipid/cholesterol metabolism.

4.1. Plasma Lipoprotein Synthesis and Metabolism. Important organs in the body that are responsible for synthesis and transport of lipoprotein are the liver and the gut. A cystic duct brings bile from the gallbladder to the gut. The liver produces the bile, but it is moved to the gallbladder and remains there to be used. Once a fatty meal arrives at the small intestine, bile salts get into action and help with emulsification of the fats. This makes their digestion and absorption in the gut possible. Fatty acids, triglycerides, and cholesterol combine in the epithelial cells of the gut where they are covered with a layer of protein. These are called chylomicrons [88]. The lymphatic system absorbs these chylomicrons and later releases them into the blood. Chylomicrons find their way to the liver and it turns them into triglyceride and cholesterol. Bile salts do not end up in the gut with the fats. They move down all the way to the ileum, where, most of the bile salts are absorbed once again and entered into the blood. The circulation takes the bile salts back to the liver. They remain in the gallbladder, with bile to be used for the above process again. Some of the bile salts are not absorbed in the small intestine and end up in the colon and are disposed of with feces. The liver makes up for the loss of bile salts by synthesizing them from its cholesterol reservoir. Cells in the liver also synthesize cholesterol and are therefore another major source of the body's cholesterol pool, in addition to the dietary sources of cholesterol. A number of factors, such as genes and diet, modulate the liver to produce cholesterol.

4.1.1. Biosynthesis of Cholesterol. Just less than $50 \%$ of the body's cholesterol comes from new biosynthesis, nearly $10 \%$ in the liver and $15 \%$ in the intestine [88]. Cholesterol synthesis occurs in the microsomes and cytoplasm from the twocarbon acetate group of acetyl-CoA [89]. The biosynthesis of cholesterol goes through the following stages [89]:

(i) conversion of acetyl-CoAs to 3-hydroxy-3-methylglutaryl-CoA (HMG-CoA),

(ii) conversion of HMG-CoA to mevalonate,

(iii) changing of mevalonate to isopentenyl pyrophosphate,

(iv) changing of isopentenyl pyrophosphate to squalene,

(v) conversion of squalene to cholesterol.

4.1.2. Regulating Cholesterol Synthesis. In healthy adults, about 1 gram of cholesterol is synthesized and 0.3 gram is consumed per day. The body maintains a relatively constant amount of cholesterol (150-200 mg/dL). This is done mainly through controlling the level of de novo synthesis. Dietary intake of cholesterol in part regulates the level of cholesterol synthesis. Both of these cholesterols are then used in the formation of membranes and in the synthesis of the steroid hormones and bile acids [90]. Bile acid synthesis uses most of this cholesterol.

Three separate mechanisms regulate the body's constant supply of cholesterol from cells [88] as follows

(i) regulation of HMG-CoA reductase (HMGR),

(ii) regulation of extra intracellular free cholesterol via acyl-CoA cholesterol acyltransferase (ACAT),

(iii) regulation of cholesterol levels in plasma via HDLmediated reverse transport and LDL receptor-mediated uptake,

The cholesterol pool of the liver is used in two important ways. The liver utilizes part of it to produce bile salts, to be stored in the gallbladder as a part of the bile and ends up in the gut. There, the bile salts are involved in the emulsification of fats and their ingestion and absorption. The rest of the cholesterol is used for other requirements of the body. To do this, the liver combines cholesterol from its pool with triglycerides and covers it with a particular protein so that it could be dissolved in the blood. These are somewhat large molecules, known as VLDL (very-low-density lipoproteins). The liver then drains them into the blood. Lipoprotein lipase (LPL) exists in abundance all over the body, especially in the walls of the arteries. This enzyme is involved in removing triglycerides from VLDL cholesterol. In the process, the VLDL shrinks in size and a relatively larger portion of it is made up of what is called intermediate-density lipoproteins, or IDL. 
Low-Density Lipoprotein (LDL). As the process continues and more triglycerides are taken away, what is left is a dense molecule referred to as low-density lipoprotein (LDL). This lipoprotein still maintains a large amount of cholesterol. The protein layer allows the tissues to use this cholesterol, LDL receptors on these tissues that make this interaction possible. In the tissues such as those of the liver and the inner layer of the arterial wall, cholesterol is taken away from low-density lipoproteins. Free radicals in the body are very reactive and oxidative compounds that can oxidize low-density lipoprotein cholesterol and help atherosclerotic plaque to form in the arteries. Antioxidants in the body can inhibit this process [91].

High-Density Lipoprotein (HDL). The liver also produces another type of lipoprotein, named high-density lipoprotein. This is different from VLDL, which is also produced in the liver. It has little triglyceride and cholesterol and has a particular protein covering. High-density lipoprotein collects the surplus cholesterol that cholesterol metabolizing cells cannot utilize. Lecithin-cholesterol acyltransferase is an enzyme that is responsible for transporting surplus cholesterol back to HDL molecules. Unused cholesterol from arteries, liver, and other tissues is absorbed by HDL cholesterol. There is evidence that even some oxidized LDL can be removed by the LCAT and HDL cholesterol [92]. As HDL circulates in the body and collects the cholesterol from tissues, it becomes mature and goes back to the liver. There, it is identified by its lipoprotein covering and is lodged in the liver's cholesterol pool.

Apo-A-1. Apo-A-1 is the main apolipoprotein in HDL cholesterol and performs a key function of collecting surplus cholesterol from the outer cells and transporting it back to the liver. It also has antioxidant and anti-inflammatory properties [93].

Apo33-B/Apo-A ratio is an indicator of cardiovascular risk. The higher the ratio, the higher the probability of cholesterol deposits in the walls of the arteries [94].

Apo-B. Apo-B is found in all of the atherogenic particles; VLDL, IDL, as well as large and small dense LDL cholesterol. They all have one Apo-B molecule inside them. The number of Apo-B, therefore, is an indicator of the number of the above particles. Apo-B helps to capture these particles from the walls of the arteries. On the other hand, the Apo-B formed in the liver helps with stabilization and transfer of cholesterol and triglycerides in plasma IDL, VLDL, and sdLDL, and with the collecting of cholesterol in the liver and the outer tissues. Of all the Apo-B particles in the blood, over ninety percent are in low-density-lipid cholesterol. Low-to-normal LDL cholesterol may indicate an increase in highly atherogenic sd-LDL particles that are readily oxidized, leading to increased formation of plaques on the arteries walls. Apo-B/Apo-A ratio is an indicator of cardiovascular risk. The higher the ratio, the higher the probability of cholesterol deposits in the walls of the arteries [94].
4.2. Probiotics' Mechanism of Action on Lipids. It has been proposed that, when probiotics settle in the gut, they ferment indigestible carbohydrate from food. Their action raises the short-chain fatty acids (SCFAs) in the gut [86]. SCFAs are produced from peptide, polysaccharide, protein, and oligosaccharide, mainly by anaerobic bacteria, and are the final product of bacteria's activity into the GI tract. In terms of quantity, carbohydrates are the main source of short-chain fatty acids [86]. These large molecules get depolymerised by a variety of hydrolytic enzymes that are produced by bacteria and allow the organisms to ferment their sugar content. SCFA can lower the lipids in blood through blocking synthesis of hepatic cholesterol and/or through redirecting plasma cholesterol toward the liver [95]. A hundred to $450 \mathrm{mmol}$ of the SFCA is produced in the large intestine every day with relative proportions of acetate, propionate, and butyrate being about $60: 20: 15$ depending on the substrate [86]. While acetate seems to increase total cholesterol, propionate increases glucose in the blood and reduces hypercholesterolemia response caused by acetate. Propionate does that by decreasing its use by the liver, for cholesterol and fatty acids synthesis. In addition, SCFAs are potential modulator of food intake and energy sensing process into the brain, which might indirectly play an important role in reduction of cholesterol and other metabolism deranging lipids into the host body [96]. Micelles, which play a role in the absorption of cholesterol in the intestine, are produced by bile salts, cholesterol, and phospholipids. By producing bile acids through deconjugating the bile salts in the small intestine, probiotics prevent micelle production. When cholesterol enters the enterohepatic circulation, it is death in the same way. Probiotics by using hydroxysteroid dehydrogenase, and conjugated bile acid hydrolase enzymes, breakdown the bile acid and hydrolyze bile salts. By doing so bile acids' enterohepatic circulation will be disrupted [9799]. Hydroxymethylglutarate CoA (HMG CoA) is another compound that helps probiotics block HMG-CoA reductase activity, which is a rate-limiting enzyme and is involved in endogenous production of cholesterol. Probiotic bacteria reduce absorption of cholesterol in the intestine by binding and hence incorporating it to the cell membrane. Cholesterol can also be assimilated during growth [100]. All of the abovementioned activities together help with the cholesterollowering actions of probiotics.

4.3. Mechanisms of Cholesterol-Lowering Effects. Past in vitro studies have evaluated a number of mechanisms proposed for the cholesterol-lowering effects of probiotics and prebiotics. One of the purported mechanisms includes enzymatic deconjugation of bile acids by bile salt hydrolase of probiotics. Bile, a water-soluble end product of cholesterol in the liver, is stored and concentrated in the gallbladder and released into the duodenum upon ingestion of food [101]. It consists of cholesterol, phospholipids, conjugated bile acids, bile pigments and electrolytes. Once deconjugated, bile acids are less soluble and absorbed by the intestines, leading to their elimination in the feces. Cholesterol is used to synthesize new bile acids in a homeostatic response, 
resulting in lowering of serum cholesterol [101] (Figure 1). In an in vitro study, Jones et al. [102] evaluated the role of bile salt hydrolase in cholesterol lowering using Lactobacillus plantarum. The authors found that BSH activity was able to hydrolyze conjugated glycodeoxycholic acid and taurodeoxycholic acid, leading to the deconjugation of glycoand taurobile acids. The hypocholesterolemic effect of the probiotics has also been attributed to their ability to bind cholesterol in the small intestines. Usman [27] previously reported that strains of Lactobacillus gasseri could remove cholesterol from laboratory media via binding onto cellular surfaces. The ability of cholesterol binding appeared to be growth and strain specific. Kimoto et al. [103] later strengthened such a hypothesis by evaluating the removal of cholesterol by probiotics cells during different growth conditions. Live and growing cells were compared to those that were nongrowing (live but suspended in phosphate buffer) and dead (heat-killed). It was observed that, although growing cells removed more cholesterol than dead cells, the heat-killed cells could still remove cholesterol from media, indicating that some cholesterol was bound to the cellular surface. Cholesterol was also removed by probiotics by incorporation into the cellular membranes during growth. Kimoto et al. [103] have examined the removal of cholesterol by several strains of lactococci from media. A difference in the fatty acid distribution pattern was observed for cells grown in the presence and absence of cholesterol. Lipids of probiotics are predominantly found in the membrane, suggesting that cholesterol incorporated into the cellular membrane had altered the fatty acid composition of the cells. The incorporation of cholesterol into the cellular membrane increased the concentration of saturated and unsaturated fatty acids, leading to increased membrane strength and subsequently higher cellular resistance toward lysis [104, 105]. Lye et al. [104] also further evaluated this mechanism by determining the possible locations of the incorporated cholesterol within the membrane phospholipid bilayer of probiotic cells. Fluorescence probes were incorporated into the membrane bilayer of probiotic cells that were grown in the absence and presence of cholesterol. Enrichment of cholesterol was found in the regions of the phospholipid tails, upper phospholipids, and polar heads of the cellular membrane phospholipid bilayer in cells that were grown in the presence of cholesterol compared to the control cells, indicating incorporation of cholesterol in those regions. Cholesterol can also be converted in the intestines to coprostanol, which is directly excreted in feces. This decreases the amount of cholesterol being absorbed, leading to a reduced concentration in the physiological cholesterol pool. Possible conversion of cholesterol into coprostanol by bacteria has been evaluated by Chiang et al. [106]. In their study, it was found that cholesterol dehydrogenase/isomerase produced by bacteria such as Sterolibacterium denitrificans was responsible for catalyzing the transformation of cholesterol to cholest-4en-3-one, an intermediate cofactor in the conversion of cholesterol to coprostanol. This served as a fundamental for further evaluations using strains of probiotic bacteria. In a recent in vitro study, Lye et al. [105] evaluated the conversion of cholesterol to coprostanol by strains of lactobacilli such as Lactobacillus acidophilus, L. bulgaricus, and L. casei ATCC 393 via fluorometric assays. The authors detected both intracellular and extracellular cholesterol reductase in all strains of probiotics examined, indicating possible intracellular and extracellular conversion of cholesterol to coprostanol. The concentration of cholesterol in the medium also decreased upon fermentation by probiotics accompanied by increased concentrations of coprostanol. This mechanism warrants further evaluations as cholesterol reductase is also directly administered to humans to convert cholesterol to coprostanol in the small intestines for a bloodstream cholesterol-lowering effect. Most of the hypotheses raised to date are based on in vitro experiments, and few attempts have been made to evaluate the possible hypocholesterolemic mechanisms based on in vivo trials. Most of the in vivo trials conducted thus far have focused heavily on verifying the hypocholesterolemic effects of probiotics, rather than the mechanisms involved. Liong et al. [107] had evaluated the hypocholesterolemic effect of a synbiotic and the possible mechanisms involved by using hypercholesterolemic pigs. In their parallel 8-week study, the authors found that the administration of a synbiotic containing L. acidophilus ATCC 4962, fructooligosaccharides, inulin, and mannitol decreased plasma total cholesterol, LDL-cholesterol, and triacylglycerols compared to the control. These lipoproteins were subsequently subfractionated and characterized. Pigs supplemented with the synbiotic had a lower concentration of cholesteryl esters in the LDL particles, accompanied by a higher concentration of triacylglycerol. Triacylglycerolenriched LDL particles are more susceptible to hydrolysis and removal from blood, while loss of cholesteryl esters forms smaller and denser LDL particles leading to a higher removal from blood compared to larger LDL particles. The authors also found that the administration of the synbiotic led to higher concentration of cholesteryl esters in the HDL particles. HDL is termed as the beneficial cholesterol attributed to its role of transporting cholesterol to the liver for further hydrolysis. Cholesterol is transported as cholesteryl esters in the core of HDL. Thus, it was suggested that the synbiotic induced a hypocholesterolemic effect via altering the pathways of cholesteryl esters and lipoprotein transporters. Prebiotics such as inulin and fructooligosaccharides are soluble, indigestible, viscous, and fermentable compounds that contribute to hypocholesterolemia via two mechanisms: decreasing cholesterol absorption accompanied by enhanced cholesterol excretion via feces and the production of short-chain fatty acids (SCFAs) upon selective fermentation by intestinal bacterial microflora (Figure 2) [108]. Using hypercholesterolemic-induced rats, Kim and Shin [109] also found that the administration of inulin for 4-weeks decreased serum LDL-cholesterol with increased serum HDL-cholesterol levels $(P<0.05)$ compared to the control. Rats fed with inulin also showed higher excretions of fecal lipid and cholesterol compared to the control $(P<$ $0.05)$, mainly attributed to reduced cholesterol absorption. Similar to indigestible fibers, soluble indigestible prebiotics have been postulated to increase the viscosity of the digestive tract and increase the thickness of the unstirred layer in the small intestine and thus inhibiting the uptake of cholesterol 
[110]. This may have led to a higher cholesterol catabolism in the liver that contributed to a hypocholesterolemic effect.

\section{Conclusion and Future Prospects}

Probiotics have received much attention on their proclaimed health benefits which include improvement in lactose intolerance, increase in natural resistance to infectious disease in gastrointestinal tract, suppression of cancer, reduction in serum cholesterol level, and improved digestion. In addition, there has been considerable interest in the effect of probiotics on human lipid metabolism, and numerous studies have focused on the potential hypocholesterolemic activity of probiotics in human. Despite these claimed benefits from the human clinical studies carried out for the last two decades, a decisive outcome has failed to be reached due to controversies raised. Also, the exact mechanism for cholesterol removal is poorly understood. Several possible mechanisms for cholesterol removal by probiotics have been proposed including assimilation of cholesterol by growing cells, binding of cholesterol to cellular surface, incorporation of cholesterol into the cellular membrane, deconjugation of bile via bile salt hydrolase, and coprecipitation of cholesterol with deconjugated bile; however, some of these mechanisms are strain dependent, and conditions generated under laboratory conditions would not be practical in the in vivo systems. Such discrepancies in the data of different effects on serum cholesterol levels may come from the differences in genus, species, and strains of lactic acid bacteria. Even though the hypocholesterolemic mechanism of probiotics has not yet been fully understood, it is an established fact that cholesterol and bile salt metabolism are closely linked. Recently "BSH hypothesis" has being proposed to explain cholesterol-lowering effects of probiotics. More recently, the hypocholesterolemic effects of some probiotics, which showed high BSH activities from in vitro trials, have been confirmed in human as well as in animals. However, the hypocholesterolemic mechanism of probiotics based on the BSH hypothesis has not yet been sufficiently elucidated. Moreover, considering that a number of commercial probiotic strains exhibit high BSH activities, further studies are needed to determine whether the BSH activity of the probiotics strains is beneficial or detrimental to the host. In probiotic research, bile tolerance is considered of primary importance in the selection of strains as bile tolerance enables the bacteria to survive its transit along the duodenum and subsequently to grow and colonize the gut epithelia. Thus, it is important to understand the physiological and molecular mechanisms by which enteric microorganisms including bifidobacteria have evolved to resist against antimicrobial activity of bile in the GI tract. Further investigation on the conserved and variable regions of the $b s h$ genes from various species could be useful for the development of alternative phylogenetic marker for bifidobacteria. Furthermore, one of the future challenges will be to unravel the physiological impacts of bile salt hydrolase activity on the enzymeproducing bacterial and mammalian cells.

\section{References}

[1] H. Aloğlu and Z. Öner, "Assimilation of cholesterol in broth, cream, and butter by probiotic bacteria," European Journal of Lipid Science and Technology, vol. 108, no. 9, pp. 709-713, 2006.

[2] WHO, "Cardiovascular Disease," Fact sheet no. 317, WHO, Geneva, Switzerland, 2009, http://www.who.int/mediacentre/factsheets/fs317/en/print.html.

[3] P. S. Yusuf, S. Hawken, S. Ônpuu et al., "Effect of potentially modifiable risk factors associated with myocardial infarction in 52 countries (the INTERHEART study): case-control study," Lancet, vol. 364, no. 9438, pp. 937-952, 2004.

[4] WHO, "Diet, Nutrition and Prevention of Chronic Diseases," Report of a Joint WHO/FAO Expert Consultation, Geneva, Switzerland, 2003.

[5] S. Dunn-Emke, G. Weidner, and D. Ornish, "Benefits of a low-fat plant-based diet," Obesity Research, vol. 9, no. 11, p. $731,2001$.

[6] E. G. Bliznakov, "Lipid-lowering drugs (statins), cholesterol, and coenzyme Q10. The Baycol case-a modern Pandora's box," Biomedicine and Pharmacotherapy, vol. 56, no. 1, pp. 56-59, 2002.

[7] I. De Smet, P. De Boever, and W. Verstraete, "Cholesterol lowering in pigs through enhanced bacterial bile salt hydrolase activity," British Journal of Nutrition, vol. 79, no. 2, pp. 185194, 1998.

[8] I. De Smet, L. Van Hoorde, M. Vande Woestyne, H. Christiaens, and W. Verstraete, "Significance of bile salt hydrolytic activities of lactobacilli," Journal of Applied Bacteriology, vol. 79, no. 3, pp. 292-301, 1995.

[9] T. A. B. Sanders, "Food production and food safety," British Medical Journal, vol. 318, no. 7199, pp. 1689-1693, 1999.

[10] R. Nagpal, H. Yadav, A. K. Puniya, K. Singh, S. Jain, and F. Marotta, "Potential of probiotics and prebiotics for synbiotic functional dairy foods," International Journal of Probiotics and Prebiotics, vol. 2, pp. 75-84, 2007.

[11] M. Kumar, P. V. Behare, D. Mohania, S. Arora, A. Kaur, and R. Nagpal, "Lactobacillus acidophilus 74-2 and Bifidobacterium animalis subsp lactis DGCC 420 modulate unspecific cellular immune response in healthy adults," European Journal of Clinical Nutrition, vol. 62, no. 5, pp. 584-593, 2008.

[12] S. Bengmark, S. Ahrne, G. Molin, and B. Jeppsson, "Intestinal colonizing lactobacilli," Journal of Nutrition, vol. 14, no. 8, pp. 585-594, 1998.

[13] D. R. Mack, S. Michail, S. Wei, L. McDougall, and M. A. Hollingsworth, "Probiotics inhibit enteropathogenic E. coli adherence in vitro by inducing intestinal mucin gene expression," American Journal of Physiology, vol. 276, no. 4, pp. G941-G950, 1999.

[14] H. Yadav, S. Jain, and P. R. Sinha, "Antidiabetic effect of probiotic dahi containing Lactobacillus acidophilus and Lactobacillus casei in high fructose fed rats," Nutrition, vol. 23, no. 1, pp. 62-68, 2007.

[15] H. Yadav, S. Jain, and P. R. Sinha, "Formation of oligosaccharides in skim milk fermented with mixed dahi cultures, Lactococcus lactis ssp diacetylactis and probiotic strains of lactobacilli," Journal of Dairy Research, vol. 74, no. 2, pp. 154159, 2007.

[16] M. Kumar, D. Mohania, D. Poddar et al., "A probiotic fermented milk prepared by mixed culture reduces pathogen shedding and alleviates disease signs in rats challenged with pathogens," International Journal of Probiotics and Prebiotics, vol. 4, no. 3, pp. 211-217, 2009. 
[17] M. Kumar, A. Kumar, R. Nagpal et al., "Cancer-preventing attributes of probiotics: An update," International Journal of Food Sciences and Nutrition, vol. 61, no. 5, pp. 473-496, 2010.

[18] M. Kumar, V. Verma, R. Nagpal et al., "Anticarcinogenic effect of probiotic fermented milk and Chlorophyllin on aflatoxin-B1 induced liver carcinogenesis in rats," British Journal of Nutrition, vol. 107, pp. 1006-1016, 2012.

[19] M. Kumar, V. Verma, R. Nagpal et al., "Effect of probiotic fermented milk and chlorophyllin on gene expressions and genotoxicity during AFB1-induced hepatocellular carcinoma," Gene, vol. 490, no. 1-2, pp. 54-59, 2011.

[20] K. Niedzielin, H. Kordecki, and B. Birkenfeld, "A controlled, double-blind, randomized study on the efficacy of Lactobacillus plantarum $299 \mathrm{~V}$ in patients with irritable bowel syndrome," European Journal of Gastroenterology and Hepatology, vol. 13, no. 10, pp. 1143-1147, 2001.

[21] T. A. B. Sanders, "Food production and food safety," British Medical Journal, vol. 318, no. 7199, pp. 1689-1693, 1999.

[22] R. Nagpal, P. V. Behare, M. Kumar et al., "Milk, milk products and disease free health: an updated overview," Critical Reviews in Food Science and Nutrition, vol. 52, no. 4, pp. 1549-7852, 2011.

[23] D. I. A. Pereira and G. R. Gibson, "Effects of consumption of probiotics and prebiotics on serum lipid levels in humans," Critical Reviews in Biochemistry and Molecular Biology, vol. 37, no. 4, pp. 259-281, 2002.

[24] F. A. M. Klaver and R. Van der Meer, "The assumed assimilation of cholesterol by lactobacilli and Bifidobacterium bifidum is due to their bile salt-deconjugating activity," Applied and Environmental Microbiology, vol. 59, no. 4, pp. 1120-1124, 1993.

[25] K. Tahri, J. P. Grill, and F. Schneider, "Bifidobacteria strain behavior toward cholesterol: Coprecipitation with bile salts and assimilation," Current Microbiology, vol. 33, no. 3, pp. 187-193, 1996.

[26] K. Tahri, J. P. Grill, and F. Schneider, "Involvement of trihydroxyconjugated bile salts in cholesterol assimilation by bifidobacteria," Current Microbiology, vol. 34, no. 2, pp. 7984, 1997.

[27] H. A. Usman, "Bile tolerance, taurocholate deconjugation, and binding of cholesterol by Lactobacillus gasseri strains," Journal of Dairy Science, vol. 82, no. 2, pp. 243-248, 1999.

[28] I. De Smet, L. van Hoorde, N. De Saeyer, M. Vande Woestyne, and W. Verstraete, "In vitro study of bile salt hydrolase (BSH) activity of BSH isogenic Lactobacillus plantarum 80 strains and estimation of cholesterol lowering through enhanced BSH activity," Microbial Ecology in Health and Disease, vol. 7, no. 6, pp. 315-329, 1994.

[29] B. Z. De Rodas, S. E. Gilliland, and C. V. Maxwell, "Hypocholesterolemic action of Lactobacillus acidophilus ATCC 43121 and calcium in swine with hypercholesterolemia induced by diet," Journal of Dairy Science, vol. 79, no. 12, pp. 2121-2128, 1996.

[30] M. O. Reynier, J. C. Montet, and A. Gerolami, "Comparative effects of cholic, chenodeoxycholic, and ursodeoxycholic acids on micellar solubilization and intestinal absorption of cholesterol," Journal of Lipid Research, vol. 22, no. 3, pp. 467473, 1981.

[31] S. E. Gilliland, C. R. Nelson, and C. Maxwell, "Assimilation of cholesterol by Lactobacillus acidophilus," Applied and Environmental Microbiology, vol. 49, no. 2, pp. 377-381, 1985.

[32] M. Du Toit, C. M. A. P. Franz, L. M. T. Dicks et al., "Characterisation and selection of probiotic lactobacilli for a preliminary minipig feeding trial and their effect on serum cholesterol levels, faeces $\mathrm{pH}$ and faeces moisture content," International Journal of Food Microbiology, vol. 40, no. 1-2, pp. 93-104, 1998.

[33] D. K. Walker and S. E. Gilliland, "Relationship among bile tolerance, bile salt deconjugation, and assimilation of cholesterol by Lactobacillus acidophilus," Journal of Dairy Science, vol. 76, no. 4, pp. 956-961, 1993.

[34] M. Rojas, F. Ascencio, and P. L. Conway, "Purification and characterization of a surface protein from Lactobacillus fermentum 104R that binds to porcine small intestinal mucus and gastric mucin," Applied and Environmental Microbiology, vol. 68, no. 5, pp. 2330-2336, 2002.

[35] G. Reid, A. W. Bruce, N. Fraser, C. Heinemann, J. Owen, and B. Henning, "Oral probiotics can resolve urogenital infections," FEMS Immunology and Medical Microbiology, vol. 30, no. 1, pp. 49-52, 2001.

[36] C. Gusils, S. N. González, and G. Oliver, "Some probiotic properties of chicken lactobacilli," Canadian Journal of Microbiology, vol. 45, no. 12, pp. 981-987, 1999.

[37] C. Heinemann, J. E. T. van Hylckama Vlieg, D. B. Janssen, H. J. Busscher, H. C. van der Mei, and G. Reid, "Purification and characterization of a surface-binding protein from Lactobacillus fermentum RC-14 that inhibits adhesion of Enterococcus faecalis 1131," FEMS Microbiology Letters, vol. 190, no. 1, pp. 177-180, 2000.

[38] H. Tanaka, K. Doesburg, T. Iwasaki, and I. Mierau, "Screening of lactic acid bacteria for bile salt hydrolase activity," Journal of Dairy Science, vol. 82, no. 12, pp. 2530-2535, 1999.

[39] H. Tanaka, H. Hashiba, J. Kok, and I. Mierau, "Bile salt hydrolase of Bifidobacterium longum-biochemical and genetic characterization," Applied and Environmental Microbiology, vol. 66, no. 6, pp. 2502-2512, 2000.

[40] J. P. Grill, C. Cayuela, J. M. Antoine, and F. Schneider, "Isolation and characterization of a Lactobacillus amylovorus mutant depleted in conjugated bile salt hydrolase activity: relation between activity and bile salt resistance," Journal of Applied Microbiology, vol. 89, no. 4, pp. 553-563, 2000.

[41] B. J. B. Wood and W. H. Holzapfel, The Genera of Lactic Acid Bacteria, Blackie Academy, London, UK, 1995.

[42] G. Corzo and S. E. Gilliland, "Bile salt hydrolase activity of three strains of Lactobacillus acidophilus," Journal of Dairy Science, vol. 82, no. 3, pp. 472-480, 1999.

[43] G. Corzo and S. E. Gilliland, "Measurement of bile salt hydrolase activity from Lactobacillus acidophilus based on disappearance of conjugated bile salts," Journal of Dairy Science, vol. 82, no. 3, pp. 466-471, 1999.

[44] S. A. Moser and D. C. Savage, "Bile salt hydrolase activity and resistance to toxicity of conjugated bile salts are unrelated properties in lactobacilli," Applied and Environmental Microbiology, vol. 67, no. 8, pp. 3476-3480, 2001.

[45] G. V. Mann and A. Spoerry, "Studies of a surfactant and cholesteremia in the Maasai," American Journal of Clinical Nutrition, vol. 27, no. 5, pp. 464-469, 1974.

[46] G. V. Mann, "A factor in yoghurt which lowers cholesterolemia in man," Arthrosclerosis, vol. 26, pp. 335-340, 1977.

[47] M. Y. Lin and T. W. Chen, "Reduction of Cholesterol by Lactobacillus acidophilus in Culture Broth," Journal of Food and Drug Analysis, vol. 8, no. 2, pp. 97-102, 2000.

[48] K. K. Grunewald, "Serum cholesterol levels in rats fed skim milk fermented by Lactobacillus acidophilus," Journal of Food Science, vol. 47, pp. 2078-2079, 1982.

[49] M. P. Taranto, M. Medici, G. Perdigon, A. P. Ruiz Holgado, and G. F. Valdez, "Evidence for hypocholesterolemic effect of 
Lactobacillus reuteri in hypercholesterolemic mice," Journal of Dairy Science, vol. 81, no. 9, pp. 2336-2340, 1998.

[50] J. Z. Xiao, S. Kondo, N. Takahashi et al., "Effects of milk products fermented by Bifidobacterium longum on blood lipids in rats and healthy adult male volunteers," Journal of Dairy Science, vol. 86, no. 7, pp. 2452-2461, 2003.

[51] I. A. Abd El-Gawad, E. M. El-Sayed, S. A. Hafez, H. M. ElZeini, and F. A. Saleh, "The hypocholesterolaemic effect of milk yoghurt and soy-yoghurt containing bifidobacteria in rats fed on a cholesterol-enriched diet," International Dairy Journal, vol. 15, no. 1, pp. 37-44, 2005.

[52] R. Kumar, S. Grover, and V. K. Batish, "Hypocholesterolaemic effect of dietary inclusion of two putative probiotic bile salt hydrolase-producing Lactobacillus plantarum strains in Sprague-Dawley rats," British Journal of Nutrition, pp. 112,2010

[53] S. Y. Lin, J. W. Ayres, W. Winkler, and W. E. Sandine, "Lactobacillus effects on cholesterol: in vitro and in vivo results," Journal of Dairy Science, vol. 72, no. 11, pp. 28852899, 1989.

[54] J. C. Mohan, R. Arora, and M. Khalilullah, "Preliminary observations on effect of Lactobacillus sporogenes on serum lipid levels in hypercholesterolemic patients," Indian Journal of Medical Research Section B, vol. 92, pp. 431-432, 1990.

[55] F. D. Gilliland, R. Mahler, W. C. Hunt, and S. M. Davis, "Preventive health care among rural American Indians in New Mexico," Preventive Medicine, vol. 28, no. 2, pp. 194202, 1999.

[56] A. Klein, U. Friedrich, H. Vogelsang, and G. Jahreis, "Lactobacillus acidophilus 74-2 and Bifidobacterium animalis subsp lactis DGCC 420 modulate unspecific cellular immune response in healthy adults," European Journal of Clinical Nutrition, vol. 62, no. 5, pp. 584-593, 2008.

[57] M. L. Jones, C. J. Martoni, M. Parent, and S. Prakash, "Cholesterol-lowering efficacy of a microencapsulated bile salt hydrolase-active Lactobacillus reuteri NCIMB 30242 yoghurt formulation in hypercholesterolaemic adults," British Journal of Nutrition, vol. 9, pp. 1-9, 2011.

[58] M. C. Carey and W. C. Duane, "Enterohepatic circulation," in The Liver: Biology and Pathobiology, I. M. Arias, N. Boyer, N. Fausto, W. B. Jackoby, D. A. Schachter, and D. A. Shafritz, Eds., pp. 719-738, Raven Press, Ltd., New York, NY, USA, 1994.

[59] A. F. Hofmann, "Bile acids," in The Liver: Biology and Pathobiology, I. M. Arias, J. L. Boyer, N. Fausto, W. B. Jackoby, D. A. Schachter, and D. A. Shafritz, Eds., pp. 677-718, Raven Press, New York, NY, USA, 1994.

[60] M. Begley, C. G. M. Gahan, and C. Hill, "The interaction between bacteria and bile," FEMS Microbiology Reviews, vol. 29, no. 4, pp. 625-651, 2005.

[61] M. Begley, R. D. Sleator, C. G. M. Gahan, and C. Hill, "Contribution of three bile-associated loci, bsh, pva, and $b t l B$, to gastrointestinal persistence and bile tolerance of Listeria monocytogenes," Infection and Immunity, vol. 73, no. 2, pp. 894-904, 2005.

[62] Z. R. Vlahcevic, D. M. Heuman, and P. B. Hylemon, "Physiology and pathophysiology of enterohepatic circulation of bile acids," in Hepatology: A Textbook of Liver Disease, D. Zakim and T. Boyer, Eds., pp. 376-417, Saunders, Philadelphia, Pa, USA, 3rd edition, 1996.

[63] O. Bortolini, A. Medici, and S. Poli, "Biotransformations on steroid nucleus of bile acids," Steroids, vol. 62, no. 8-9, pp. 564-577, 1997.
[64] A. K. Batta, G. Salen, R. Arora, S. Shefer, M. Batta, and A. Person, "Side chain conjugation prevents bacterial 7dehydroxylation of bile acids," Journal of Biological Chemistry, vol. 265, no. 19, pp. 10925-10928, 1990.

[65] C. A. Elkins, S. A. Moser, and D. C. Savage, "Genes encoding bile salt hydrolases and conjugated bile salt transporters in Lactobacillus johnsonii 100-100 and other Lactobacillus species," Microbiology, vol. 147, no. 12, pp. 3403-3412, 2001.

[66] C. M. A. P. Franz, I. Specht, P. Haberer, and W. H. Holzapfel, "Bile salt hydrolase activity of enterococci isolated from food: screening and quantitative determination," Journal of Food Protection, vol. 64, no. 5, pp. 725-729, 2001.

[67] D. R. Edgell, M. Belfort, and D. A. Shub, "Barriers to intron promiscuity in bacteria," Journal of Bacteriology, vol. 182, no. 19, pp. 5281-5289, 2000.

[68] O. McAuliffe, R. J. Cano, and T. R. Klaenhammer, "Genetic analysis of two bile salt hydrolase activities in Lactobacillus acidophilus NCFM," Applied and Environmental Microbiology, vol. 71, no. 8, pp. 4925-4929, 2005.

[69] S. M. Huijghebaert, J. A. Mertens, and H. J. Eyssen, "Isolation of a bile salt sulfatase producing Clostridium strain from rat intestinal microflora," Applied and Environmental Microbiology, vol. 43, no. 1, pp. 185-192, 1982.

[70] J. Van Eldere, P. Celis, G. De Pauw, E. Lesaffre, and H. Eyssen, "Tauroconjugation of cholic acid stimulates $7 \alpha-$ dehydroxylation by fecal bacteria," Applied and Environmental Microbiology, vol. 62, no. 2, pp. 656-661, 1996.

[71] G. W. Tannock, M. P. Dashkevicz, and S. D. Feighner, "Lactobacilli and bile salt hydrolase in the murine intestinal tract," Applied and Environmental Microbiology, vol. 55, no. 7, pp. 1848-1851, 1989.

[72] S. E. Gilliland and M. L. Speck, "Deconjugation of bile acids by intestinal lactobacilli," Applied and Environmental Microbiology, vol. 33, no. 1, pp. 15-18, 1977.

[73] P. C. Dambekodi and S. E. Gilliland, "Incorporation of cholesterol into the cellular membrane of Bifidobacterium longum," Journal of Dairy Science, vol. 81, no. 7, pp. 18181824, 1998.

[74] M. P. Taranto, F. Sesma, A. Pesce De Ruiz Holgado, and G. F. De Valdez, "Bile salts hydrolase plays a key role on cholesterol removal by Lactobacillus reuteri," Biotechnology Letters, vol. 19, no. 9, pp. 845-847, 1997.

[75] M. P. Taranto, M. L. Fernandez Murga, G. Lorca, and G. F. De Valdez, "Bile salts and cholesterol induce changes in the lipid cell membrane of Lactobacillus reuteri," Journal of Applied Microbiology, vol. 95, no. 1, pp. 86-91, 2003.

[76] J. M. Boggs, "Lipid intermolecular hydrogen bonding: influence on structural organization and membrane function," Biochimica et Biophysica Acta, vol. 906, no. 3, pp. 353-404, 1987.

[77] I. De Smet, L. Van Hoorde, M. Vande Woestyne, H. Christiaens, and W. Verstraete, "Significance of bile salt hydrolytic activities of lactobacilli," Journal of Applied Bacteriology, vol. 79, no. 3, pp. 292-301, 1995.

[78] J. P. Coleman and L. L. Hudson, "Cloning and characterization of a conjugated bile acid hydrolase gene from Clostridium perfringens," Applied and Environmental Microbiology, vol. 61, no. 7, pp. 2514-2520, 1995.

[79] G.-B. Kim, C. M. Miyamoto, E. A. Meighen, and B. H. Lee, "Cloning and characterization of the bile salt hydrolase genes (bsh) from Bifidobacterium bifidum strains," Applied and Environmental Microbiology, vol. 70, no. 9, pp. 56035612, 2004. 
[80] M. T. Liong and N. P. Shah, "Bile salt deconjugation ability, bile salt hydrolase activity and cholesterol co-precipitation ability of lactobacilli strains," International Dairy Journal, vol. 15, no. 4, pp. 391-398, 2005.

[81] J. M. Bateup, M. A. McConnell, H. F. Jenkinson, and G. W. Tannock, "Comparison of Lactobacillus strains with respect to bile salt hydrolase activity, colonization of the gastrointestinal tract, and growth rate of the murine host," Applied and Environmental Microbiology, vol. 61, no. 3, pp. 1147-1149, 1995.

[82] O. Dussurget, D. Cabanes, P. Dehoux et al., "Listeria monocytogenes bile salt hydrolase is a PrfA-regulated virulence factor involved in the intestinal and hepatic phases of listeriosis," Molecular Microbiology, vol. 45, no. 4, pp. 1095-1106, 2002.

[83] H. Schuster, "Improving lipid management - To titrate, combine or switch," International Journal of Clinical Practice, vol. 58, no. 7, pp. 689-694, 2004.

[84] M. P. Taranto, M. Medici, G. Perdigon, A. P. Ruiz Holgado, and G. Font de Valdez, "Effect of Lactobacillus reuteri on the prevention of hypercholesterolemia in mice," Journal of Dairy Science, vol. 83, no. 3, pp. 401-403, 2000.

[85] S. D. Feighner and M. P. Dashkevicz, "Effect of dietary carbohydrates on bacterial cholyltaurine hydrolase in poultry intestinal homogenates," Applied and Environmental Microbiology, vol. 54, no. 2, pp. 337-342, 1988.

[86] M. P. St-Onge, E. R. Farnworth, and P. J. H. Jones, "Consumption of fermented and nonfermented dairy products: Effects on cholesterol concentrations and metabolism," American Journal of Clinical Nutrition, vol. 71, no. 3, pp. 674$681,2000$.

[87] J. W. Anderson and S. E. Gilliland, "Effect of fermented milk (yogurt) containing Lactobacillus acidophilus L1 on serum cholesterol in hypercholesterolemic humans," Journal of the American College of Nutrition, vol. 18, no. 1, pp. 43-50, 1999.

[88] L. A. Kaplan and A. J. Pesse, Clinical Chemistry, Theory, Analysis, and Correlation, Mosby Company, St. Louis, Mo, USA, 3rd edition, 1996.

[89] S. Dessi and B. Batetta, "Overview-intracellular cholesterol homeostasis: old and new players," in Cell Growth and Cholesterol Esters, A. Pani and S. Dessi, Eds., pp. 1-12, Kluwer Academic/Plenum Publishers, New York, NY, USA, 2003.

[90] J. B. Croft, J. L. Cresanta, L. S. Webber et al., "Cardiovascular risk in parents of children with extreme lipoprotein cholesterol levels: the Bogalusa Heart Study," Southern Medical Journal, vol. 81, no. 3, pp. 341-349, 1988.

[91] I. Jialal, "Evolving lipoprotein risk factors: Lipoprotein(a) and oxidized low-density lipoprotein," Clinical Chemistry, vol. 44, no. 8, pp. 1827-1832, 1998.

[92] A. Höckerstedt, M. Jauhiainen, and M. J. Tikkanen, "Lecithin/cholesterol acyltransferase induces estradiol esterification in high-density lipoprotein, increasing its antioxidant potential," Journal of Clinical Endocrinology and Metabolism, vol. 89, no. 10, pp. 5088-5093, 2004.

[93] S. E. Nissen, T. Tsunoda, E. M. Tuzcu et al., "Effect of recombinant ApoA-I Milano on coronary atherosclerosis in patients with acute coronary syndromes: a randomized controlled trial," Journal of the American Medical Association, vol. 290, no. 17, pp. 2292-2300, 2003.

[94] G. Walldus, I. Jungner, A. H. Aastveit, I. Holme, C. D. Furberg, and A. D. Sniderman, "The apoB/apoA-I ratio is better than the cholesterol ratios to estimate the balance between plasma proatherogenic and antiatherogenic lipoproteins and to predict coronary risk," Clinical Chemistry and Laboratory Medicine, vol. 42, no. 12, pp. 1355-1363, 2004.
[95] V. De Preter, T. Coopmans, P. Rutgeerts, and K. Verbeke, "Influence of long-term administration of lactulose and Saccharomyces boulardii on the colonic generation of phenolic compounds in healthy human subjects," Journal of the American College of Nutrition, vol. 25, no. 6, pp. 541-549, 2006.

[96] Y. Xiong, N. Miyamoto, K. Shibata et al., "Short-chain fatty acids stimulate leptin production in adipocytes through the G protein-coupled receptor GPR41," Proceedings of the National Academy of Sciences of the United States of America, vol. 101, no. 4, pp. 1045-1050, 2004.

[97] P. De Boever and W. Verstraete, "Bile salt deconjugation by Lactobacillus plantarum 80 and its implication for bacterial toxicity," Journal of Applied Microbiology, vol. 87, no. 3, pp. 345-352, 1999.

[98] N. I. Doncheva, G. P. Antov, E. B. Softova, and Y. P. Nyagolov, "Experimental and clinical study on the hypolipidemic and antisclerotic effect of Lactobacillus bulgaricus strain GB N 1 (48)," Nutrition Research, vol. 22, no. 4, pp. 393-403, 2002.

[99] Y. T. Ahn, G. B. Kim, K. S. Lim, Y. J. Baek, and H. U. Kim, "Deconjugation of bile salts by Lactobacillus acidophilus isolates," International Dairy Journal, vol. 13, no. 4, pp. 303$311,2003$.

[100] D. O. Noh and S. E. Gilliland, "Influence of bile on cellular integrity and beta-galactosidase activity of Lactobacillus acidophilus," Journal of Dairy Science, vol. 76, no. 5, pp. 12531259, 1993.

[101] M. Begley, C. Hill, and C. G. M. Gahan, "Bile salt hydrolase activity in probiotics," Applied and Environmental Microbiology, vol. 72, no. 3, pp. 1729-1738, 2006.

[102] M. L. Jones, H. Chen, W. Ouyang, T. Metz, and S. Prakash, "Microencapsulated genetically engineered Lactobacillus plantarum 80 ( $\mathrm{pCBH} 1$ ) for bile acid deconjugation and its implication in lowering cholesterol," Journal of Biomedicine and Biotechnology, vol. 2004, no. 1, pp. 61-69, 2004.

[103] H. Kimoto, S. Ohmomo, and T. Okamoto, "Cholesterol removal from media by lactococci," Journal of Dairy Science, vol. 85, no. 12, pp. 3182-3188, 2002.

[104] H. S. Lye, G. R. Rahmat-Ali, and M. T. Liong, "Mechanisms of cholesterol removal by lactobacilli under conditions that mimic the human gastrointestinal tract," International Dairy Journal, vol. 20, no. 3, pp. 169-175, 2010.

[105] H. S. Lye, G. Rusul, and M. T. Liong, "Removal of cholesterol by lactobacilli via incorporation and conversion to coprostanol," Journal of Dairy Science, vol. 93, no. 4, pp. 1383-1392, 2010.

[106] Y. R. Chiang, W. Ismail, D. Heintz, C. Schaeffer, A. Van Dorsselaer, and G. Fuchs, "Study of anoxic and oxic cholesterol metabolism by Sterolibacterium denitrificans," Journal of Bacteriology, vol. 190, no. 3, pp. 905-914, 2008.

[107] M. T. Liong, F. R. Dunshea, and N. P. Shah, "Effects of a synbiotic containing Lactobacillus acidophilus ATCC 4962 on plasma lipid profiles and morphology of erythrocytes in hypercholesterolaemic pigs on high- and low-fat diets," British Journal of Nutrition, vol. 98, no. 4, pp. 736-744, 2007.

[108] B. H. Arjmandi, J. Craig, S. Nathani, and R. D. Reeves, "Soluble dietary fiber and cholesterol influence in vivo hepatic and intestinal cholesterol biosynthesis in rats," Journal of Nutrition, vol. 122, no. 7, pp. 1559-1565, 1992.

[109] M. Kim and H. K. Shin, "The water-soluble extract of chicory influences serum and liver lipid concentrations, cecal shortchain fatty acid concentrations and fecal lipid excretion in 
rats," Journal of Nutrition, vol. 128, no. 10, pp. 1731-1736, 1998.

[110] C. L. Dikeman, M. R. Murphy, and G. C. Fahey, "Dietary fibers affect viscosity of solutions and simulated human gastric and small intestinal digesta," Journal of Nutrition, vol. 136, no. 4, pp. 913-919, 2006. 


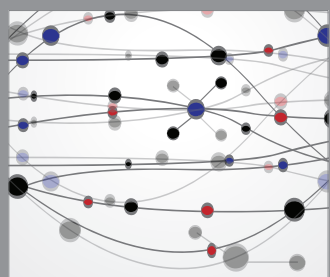

The Scientific World Journal
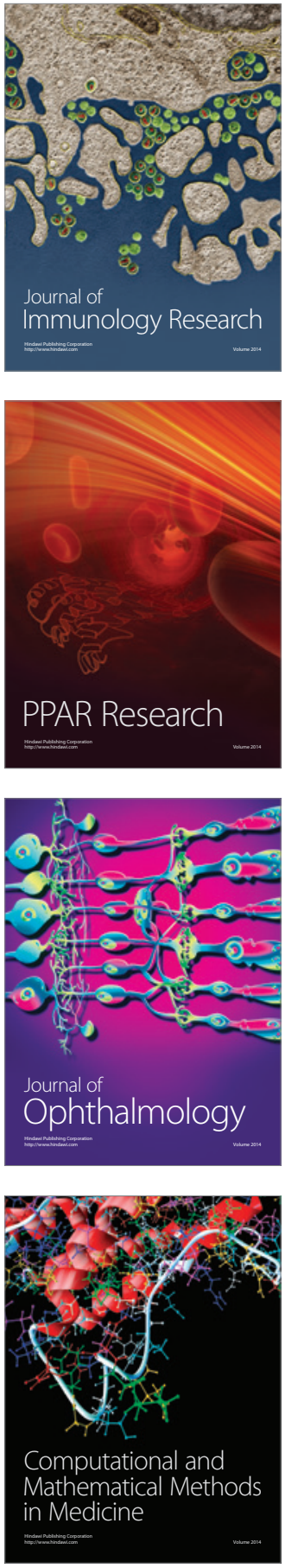

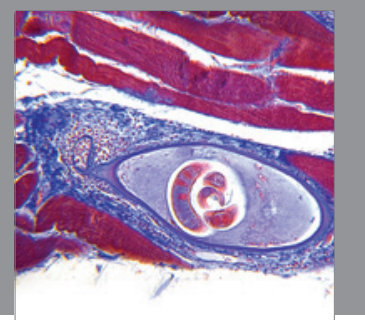

Gastroenterology

Research and Practice
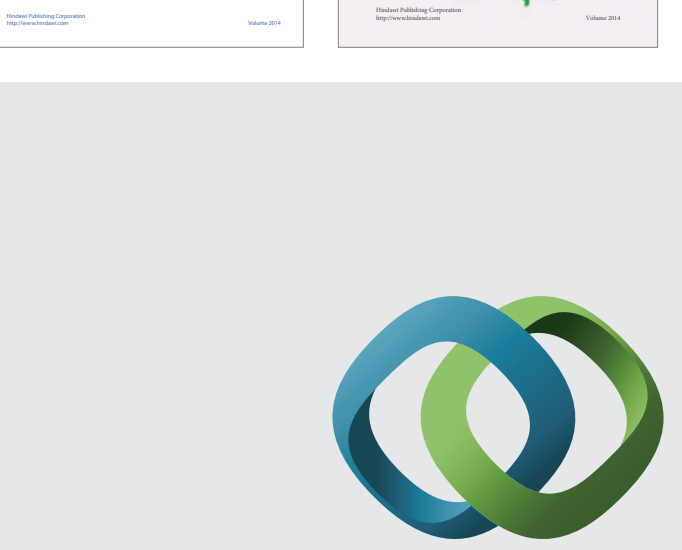

\section{Hindawi}

Submit your manuscripts at

http://www.hindawi.com
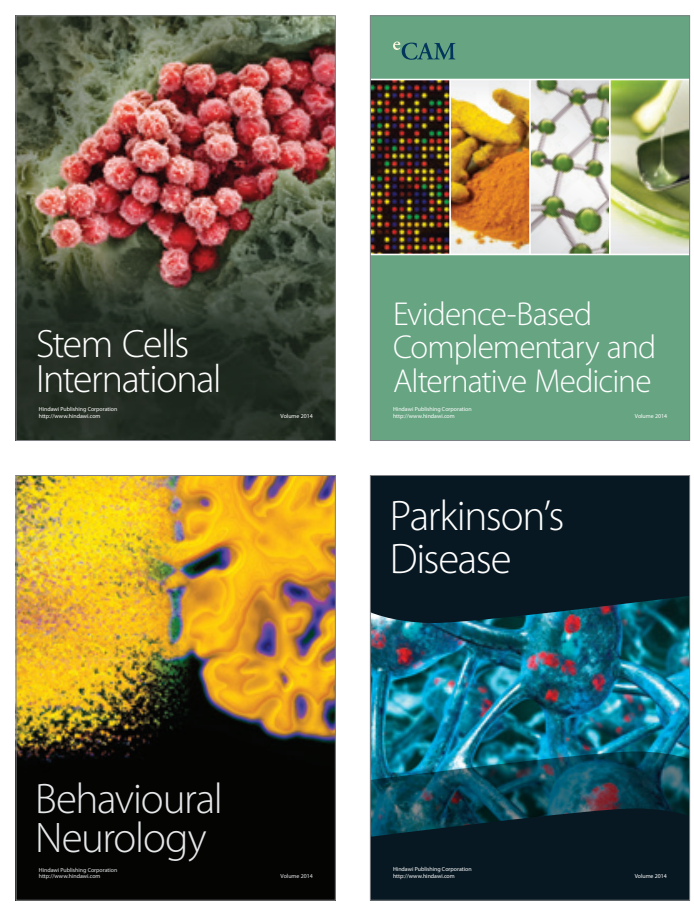

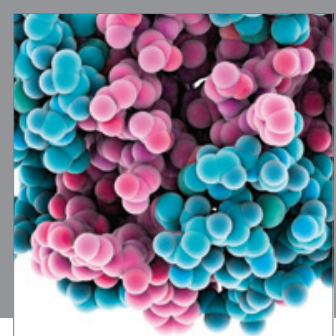

Journal of
Diabetes Research

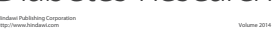

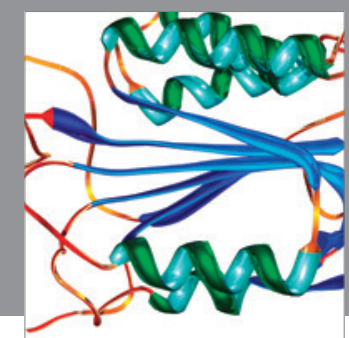

Disease Markers
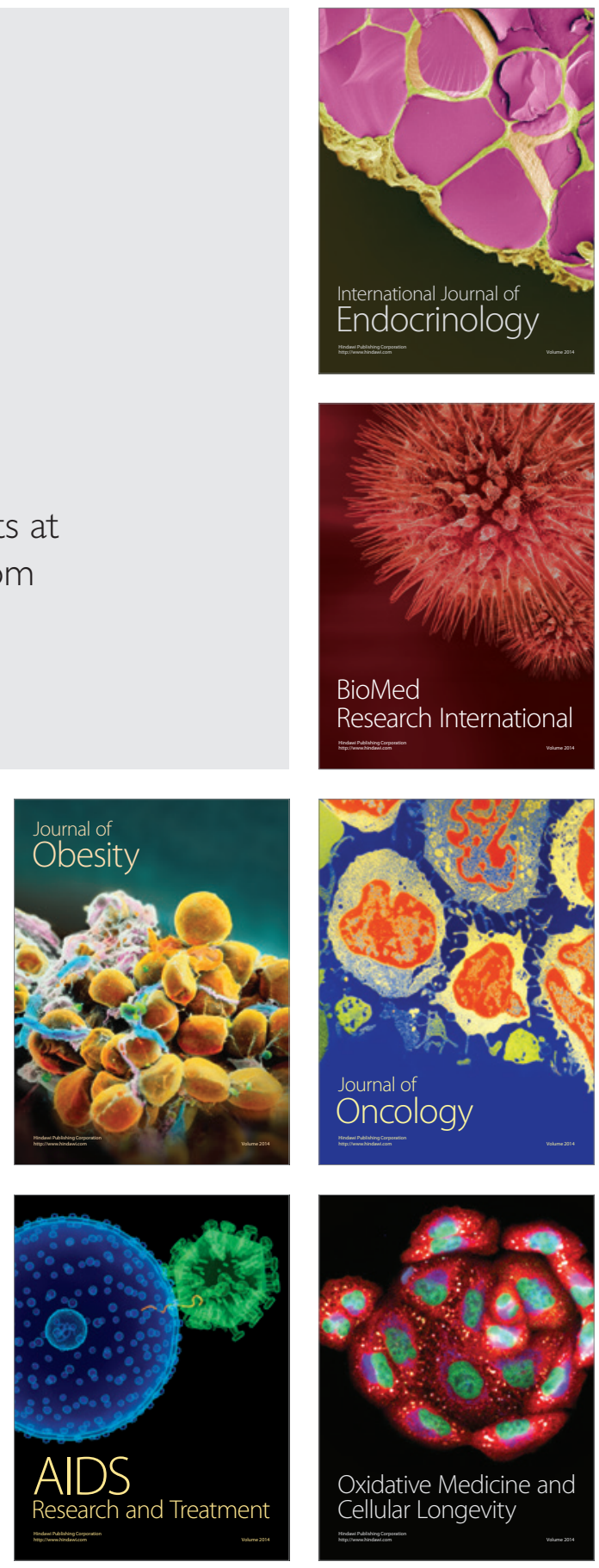